\title{
Effect on Network Performance of Common versus Private Acquisition Sequences for Impulse Radio UWB Networks
}

\author{
EPFL Technical Report LCA-REPORT-2006-005 \\ Ruben Merz, Jean-Yves Le Boudec and Saravanan Vijayakumaran \\ EPFL, School of Computer and Communication Sciences \\ \{ruben.merz, jean-yves.leboudec, saravanan.vijayakumaran\}@epfl.ch
}

The work presented in this paper was supported (in part) by the National Competence Center in Research on Mobile Information and Communication Systems (NCCR-MICS), a center supported by the Swiss National Science Foundation under grant number 5005-67322, and by CTI contract No7109.2;1 ESPP-ES 


\begin{abstract}
Packet detection and timing acquisition for IR-UWB networks such as 802.15.4a relies on the presence of an acquisition sequence (or preamble) at the beginning of each packet. A simple network design choice is to use a common acquisition sequence for the whole network. A second design choice is to use an acquisition sequence private to destinations. It potentially yields a larger network throughput, but requires additional complexity for sources to learn the acquisition sequence of their destination. In this paper, we evaluate the effect of a common or private acquisition sequence on the network throughput. Our analysis is based on analytical modeling and simulations. We show that a private acquisition sequence yields a substantial increase in throughput. The throughput difference grows with the number of concurrent transmitters and interferers. We also show the presence of a compounding effect similar to the exposed terminal issue in 802.11 networks.
\end{abstract}

\title{
I. INTRODUCTION
}

Future UWB networks will range from a few dozen nodes to large-scale networks composed of hundreds of nodes. A key ingredient for the operation of such networks is packet detection and timing acquisition. In networks such as $802.15 .4 \mathrm{a}$, or with MAC protocols for impulse-radio ultra-wide band (IR-UWB) networks like DCC-MAC [1] or $\mathrm{UWB}^{2}$ [2], packet detection and timing acquisition relies on the presence of an acquisition sequence (or acquisition preamble) at the beginning of each packet. In such cases, there is no global synchronization in the network and timing acquisition is performed on a per packet basis. One possible simple network design choice is to have an identical and common acquisition sequence for the entire network. Another proposal, as in [1], [2] is to have a private acquisition sequence per destination. In [1], [2], a source computes the acquisition sequence of its intended destination as a function of a unique identifier of the destination. Such an identifier can be, for instance, the MAC address.

With a private acquisition sequence, there is a potential throughput increase with respect to the common acquisition sequence case. Indeed, during timing acquisition, a packet might contend with only sources that intend to transmit to the same destination. In contrast, with a common acquisition sequence, contention might occur with nodes from the whole network. However, with a private acquisition sequence comes the cost of learning the acquisition sequence of the destination. Hence the throughput increase must be large (maybe $>100 \%$ ) in order to alleviate the associated cost. Note that regarding hardware implementation, a private acquisition sequence 
might not be essentially more costly since a node does not need to listen to more than a few sequences $^{1}[1]$.

In this paper, we evaluate the effect on the network throughput of having a common or private acquisition sequence. Due to the lack of space, we do not evaluate the cost of learning the acquisition sequence. It is left for further study.

We do not model packet detection and timing acquisition at the level of details of the physical layer. Due to the timescale difference between events at the physical layer and events at the link layer, the complexity would be huge. Rather, we use the probability of missed detection and the probability of false alarm derived in [3] to model packet detection and timing acquisition at the link layer level (see Section II-B).

In the case of unintentional packet acquisition (i.e. a packet not for the destination), we consider two options. With early discard, a destination drops the packet right after the header containing the hardware address. With late discard, the packet is fully received. Note that even in the case of private acquisition sequences unintentional packet acquisition can occur due to noise and multi-user interference [3].

Our performance metric is mainly the saturation throughput [4]; a source has always a packet available to transmit and queuing at the source is ignored. Even though UWB networks are expected to be low-data rate networks, the performance in saturation conditions still matters. For instance, in case of sudden bursts of activity, it is important to ensure that the network is able to sustain the sudden load.

For the evaluation, we use two different approaches. First, we derive an analytical model to compute the throughput of a UWB network in saturated conditions. Due to the inherent high difficulty, this problem is solved analytically for symmetric and homogeneous networks where all nodes are in range of each other. For simplicity, we consider noise and multi-user (MUI) interference in the analytical model only during packet detection and timing acquisition; we expect that interference in the data transmission part will have little impact on the result of our comparison, since we focus on the acquisition phase, and this is confirmed by comparison to simulation results.

Second, in order to evaluate the saturation throughput in more realistic scenarios (and to take

${ }^{1}$ Its own sequence, the one from the destination and the broadcast one. 
MUI into account during packet transmission), we turn to ns-2 [5] simulations. It also allows us to verify the results obtained with our analytical model.

We consider an ultra-wide band network with an impulse-radio physical layer. Stations use pseudo-random time-hopping sequences (THS). For packet detection and timing acquisition, we assume that there is an acquisition sequence at the beginning of each packet. Timing acquisition is done on a per packet basis. The underlying acquisition method is [3]; it is known to be robust against multi-user interference. At the MAC layer, we use the DCC-MAC protocol [1].

Our analysis can be easily conducted with a different MAC layer or a different acquisition method. Indeed, from the acquisition method, we only need the probability of missed detection and the probability of false alarm due to noise and interference.

The remaining of this paper is organized as follows. In Section II, we develop the analytical model to compute the saturation throughput of a symmetric and homogeneous UWB network. In Section III-A, we verify the accuracy of the model.

We then use the model to evaluate the performance of a symmetric and homogeneous UWB network. In Sections III-B and III-C, we consider more general scenarios using the ns-2 [5] simulator. In particular, we look at a network composed of several piconets. In this case, the throughput difference grows with the number of concurrent transmitters and interferers. We also show the presence of a compounding effect similar to exposed terminal issues in 802.11 network.

\section{A Saturation Throughrut Analysis of a UWB Network with Packet}

\section{Detection AND TIMING ACQUiSition}

In this section, we compute the saturation throughput of a UWB network. First, let $\lambda_{0}^{(i)}$ be the saturation throughput of a source $i$ in packets per second. Second, we define $\lambda^{(i)}$ to be the rate of packet transmission attempts per second. Note that $\lambda^{(i)} \geq \lambda_{0}^{(i)}$, since it comprises of successful packet transmissions and packet retransmissions. Finally, $p_{\text {acq }}^{(j)}$ is the average probability of proper packet detection and timing acquisition at a destination $j$. The total number of sources and destinations in the network is $S$ and $D$ respectively.

Generally, finding the exact saturation throughput of every source is a highly difficult problem to solve. Indeed, we have to model the interactions of each node with every other nodes. Therefore, in order to keep the analysis tractable we first make the following two assumptions 
1) The network is symmetric and homogeneous. Every destination has the same number of sources.

2) We make a mean-field assumption [6] where we assume that all sources have an identical and independent behavior. Hence $\lambda_{0}^{(i)}=\lambda_{0}$ and $\lambda^{(i)}=\lambda$ for $i=0, \ldots, S-1$, and $p_{\text {acq }}^{(j)}=p_{\text {acq }}$ for $j=0, \ldots, D-1$.

Second, we assume that in the saturated regime, the network model is ergodic. Indeed, there is no queuing and every source waits until a packet is successfully transmitted before attempting the transmission of a new packet. Therefore, there should not be any possible walk to infinity. Finally, we break our general problem into two subproblems.

1) Given a source and its intended destination, the saturation throughput $\lambda_{0}$ of the source depends on the probability of successful packet acquisition $p_{\text {acq }}$ at the destination. Hence, our first subproblem is to compute $\lambda_{0}$ (and $\lambda$ ) given $p_{\text {acq }}$ i.e. $\left[\lambda_{0}, \lambda\right]=f\left(p_{\text {acq }}\right)$. We solve this problem in Section II-A.

2) In the second subproblem we have a receiver with several sources with saturation throughput $\lambda_{0}$ and attempt rate $\lambda$. We want to compute $p_{\text {acq }}$ i.e. $p_{\text {acq }}=g\left(\lambda_{0}, \lambda\right)$. We solve this problem in Section II-B.

Hence, the saturation throughput is given by $f(x)$ where $x$ is the solution of the fixed point equation

$$
g(f(x))-x=0, \quad x \in[0,1]
$$

We solve the fixed point equation numerically.

\section{A. Computing $\lambda_{0}$ and $\lambda$ as a Function of $p_{\text {acq }}$}

1) The Retransmission Markov Chain $X_{n}$ : In this section, we solve the first subproblem. In order to compute $\lambda_{0}$ and $\lambda$, we use a discrete-time, homogeneous, Markov chain: let $X_{n}$ be the (re)transmission state of a source (see Figure 1, left) after a packet (re)transmission. Let $R$ be the maximum number of retransmissions before a packet is dropped. We then have $R+3$ states 
with the following transition probabilities:

$$
\begin{cases}p_{X}(i, i+1)=1-p_{\text {acq }}=p_{\text {fail }}, & i=0, \ldots, R \\ p_{X}(i, R+2)=p_{\text {acq }}, & i=0, \ldots, R \\ p_{X}(R+1,0)=1 & \\ p_{X}(R+2,0)=1 & \end{cases}
$$

where $p_{X}(i, j)=\mathbb{P}\left(X_{n+1}=j \mid X_{n}=i\right)$. An initial packet transmission occurs in state 0 . States $1, \ldots, R$ are the states where a packet retransmission happens. State $R+2$ is entered when a successful packet transmission occurs. State $R+1$ is the drop state. States $R+1$ and $R+2$ are not strictly necessary and could be merged with state 0 . However, they simplify the model description. The stationary distribution of $X_{n}$ is

$$
\begin{aligned}
\pi_{X}(i) & =\frac{\left(1-p_{\text {acq }}\right)^{i}}{1+\frac{1-\left(1-p_{\text {acq }}\right)^{R+1}}{p_{\text {acq }}}}, \quad i=0, \ldots, R+1 \\
\pi_{X}(R+2) & =\frac{1-\left(1-p_{\text {acq }}\right)^{R+1}}{1+\frac{1-\left(1-p_{\text {acq }}\right)^{R+1}}{p_{\text {acq }}}}
\end{aligned}
$$

where we used $\sum_{k=0}^{n} x^{k}=\frac{1-x^{n+1}}{1-x}$.

In addition to the transition probabilities, we define $m(i, j)$ the cost of a transition from state $i$ to state $j$. Let us assume that $X_{0}=0$, then $\tau_{1}=\inf \left\{n \geq 1 \mid X_{n}=0\right\}$ is the time of first return to state 0 and

$$
\mathbb{E}\left(\sum_{n=1}^{\tau_{1}} m\left(X_{n-1}, X_{n}\right) \mid X_{0}=0\right)
$$

is simply the expected cost of a trip from state 0 back to state 0 . Properly assigning costs to the transitions and using (3) is the key to compute $\lambda_{0}$ and $\lambda$. In the following two sections, we first explain how to compute (3) using results from Palm calculus theory. Then, we apply (3) to compute $\lambda_{0}$ and $\lambda$.

2) Computing the Expected Cost Using Palm Calculus:

Definition 1 (Palm probability and Palm expectation): Given an integer valued point process $T_{n}$ of rate $\lambda$, the Palm probability $\mathbb{P}^{0}$ is the conditional probability given that $T_{0}=0$. Similarly, the Palm expectation $\mathbb{E}^{0}$ is the conditional expectation given that $T_{0}=0$.

Now, let $Y_{n}$ be a discrete-time random process. We use the following result, from [7] (see also in this reference for a precise definition of joint stationarity): 
Theorem 1 (Palm inversion formula): If $T_{n}, Y_{n}$ is jointly stationary, then

$$
\mathbb{E}\left(Y_{0}\right)=\lambda \mathbb{E}^{0}\left(\sum_{s=1}^{T_{1}} Y_{s}\right)
$$

In order to compute (3), we apply Theorem 1 with $Y_{n}=m\left(X_{n-1}, X_{n}\right)$ and $T_{n}=\tau_{n}$ (the times of visit to state 0 ). Hence (3) becomes

$$
\begin{array}{r}
\mathbb{E}\left(\sum_{n=1}^{\tau_{1}} m\left(X_{n-1}, X_{n}\right) \mid X_{0}=0\right) \\
=\mathbb{E}^{0}\left(\sum_{n=1}^{T_{1}} m\left(X_{n-1}, X_{n}\right)\right)=\frac{\mathbb{E}\left(m\left(X_{n-1}, X_{n}\right)\right)}{\lambda} \\
=\frac{\sum_{i} \pi_{X}(i) \sum_{j} p_{X}(i, j) m(i, j)}{\pi_{X}(0)}
\end{array}
$$

for $i, j=0, \ldots, R+2$.

3) Using the Expected Cost to Compute $\lambda_{0}$ and $\lambda$ : We have

$$
\lambda_{0}=\frac{\mathbb{E}^{0}\left(N_{\mathrm{s}}\right)}{\mathbb{E}^{0}(T)} \quad \lambda=\frac{\mathbb{E}^{0}\left(N_{\mathrm{a}}\right)}{\mathbb{E}^{0}(T)}
$$

where, for a trip from state 0 back to state $0, T$ is the time of the trip, $N_{\mathrm{s}}$ is the number of successful packet transmissions and $N_{\mathrm{a}}$ is the number of attempted packet transmissions.

In order to obtain $\mathbb{E}^{0}\left(N_{\mathrm{s}}\right)$, we must compute (4) with the the costs

$$
m(i, R+2)=1, \quad i=0, \ldots, R
$$

and 0 otherwise. For $\mathbb{E}^{0}\left(N_{\mathrm{a}}\right)$, we use instead

$$
\begin{array}{ll}
m(i, R+2)=1, & i=0, \ldots, R \\
m(i, i+1)=1, & i=0, \ldots, R
\end{array}
$$

and 0 otherwise. Finally, $\mathbb{E}^{0}(T)$ is obtained with

$$
\begin{aligned}
& m(i, R+2)=t_{\mathrm{acq}}, \quad i=0, \ldots, R \\
& m(i, i+1)=t_{\mathrm{fail}}(i), \quad i=0, \ldots, R \\
& m(R+1,0)=t_{\mathrm{drop}} \\
& m(R+2,0)=t_{\mathrm{tx}}
\end{aligned}
$$

and 0 otherwise. Since they are protocol specific, the details of $t_{\mathrm{acq}}, t_{\text {fail }}(i), t_{\mathrm{drop}}$ and $t_{\mathrm{tx}}$ are given in Section III. Still, note that $t_{\text {fail }}(i)$ depends on $i$, i.e. it depends on the particular retransmission state; typically, as the number of retransmissions increase, the size of the contention window for the backoff timer increases. 

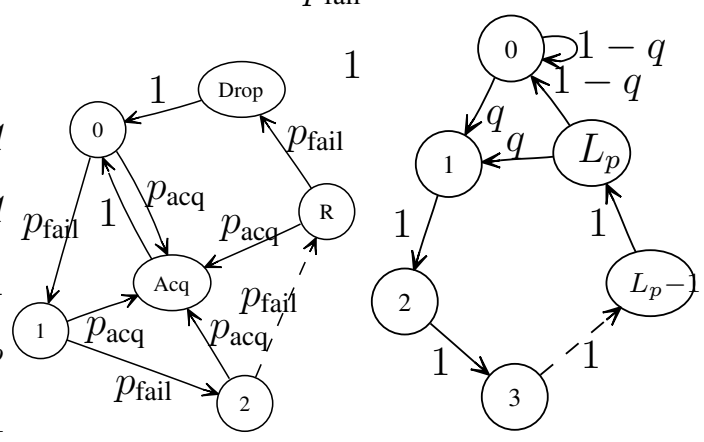

Fig. 1. Retransmission (left) Markov chain $X_{n}$ and transmission Markov chains $Z_{n}$ with their transition probabilities. Note

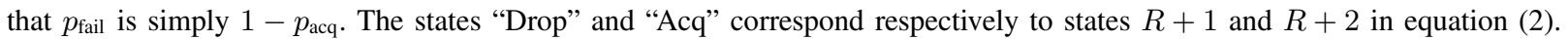

\section{B. Computing $p_{\text {acq }}$ as a Function of $\lambda_{0}$ and $\lambda$}

In this section, we solve the second subproblem. We compute $p_{\text {acq }}$ as a function of $\lambda_{0}$ and $\lambda$. Let $S_{\mathrm{D}}$ be the number of stations transmitting to the destination of interest. In addition $S_{\mathrm{I}}$ is the number of stations using the same acquisition sequence than the $S_{\mathrm{D}}$ ones but transmitting to another destination and $I$ is the number of stations using a different acquisition sequence. We have $S_{\mathrm{D}}+S_{\mathrm{I}}+I=S$. The probability of packet acquisition can be modeled as

$$
p_{\text {acq }}=\left(1-P_{\text {busy }}\right) \gamma
$$

where

$$
\begin{aligned}
\gamma & =\sum_{k=0}^{S_{\mathrm{D}}+S_{\mathrm{I}}-1} P_{k} \sum_{i=0}^{I} P_{i}\left[\frac{1}{k+1}\left(1-P_{\mathrm{MD}}^{(k, i)}\right)\right] \\
P_{k} & =\left(\begin{array}{c}
S_{\mathrm{D}}+S_{\mathrm{I}}-1 \\
k
\end{array}\right)(1-Q(l))^{k} Q(l)^{S_{\mathrm{D}}+S_{\mathrm{I}}-1-k} \\
P_{i} & =\left(\begin{array}{c}
I \\
i
\end{array}\right)(1-Q(l))^{i} Q(l)^{I-i}
\end{aligned}
$$

The quantity $P_{\mathrm{MD}}^{(k, i)}$ is the probability of missed detection given that there are $k$ concurrent transmissions with the same THS and $i$ concurrent transmissions with a different THS. In addition, $P_{\text {busy }}$ is the probability that the destination is busy (receiving a packet or transmitting an acknowledgment) and $Q(l)$ is the probability that a station does not start a packet transmission during a so-called "vulnerable period" of length $l$ chips. The vulnerable period corresponds to 
the duration of the acquisition sequence, and $l$ is the length of the acquisition sequence in chips. In the following subsections, we describe how to compute $P_{\text {busy }}$ and $Q(l)$.

1) An Expression for $P_{\text {busy }}$ in order to Compute $p_{\text {acq }}$ : The packets from any of the $S_{\mathrm{I}}$ stations can be acquired with probability $p_{\text {acq }}$ (note that it takes into account the fact that the receiver could be busy). However, for the $I$ stations with a different THS, only a fraction $P_{\mathrm{FA}}$ is acquired. Hence, we obtain

$$
P_{\text {busy }}=\lambda_{0}\left(S_{\mathrm{D}}-1\right) t_{\mathrm{D}}+\lambda\left[p_{\mathrm{acq}} S_{\mathrm{I}}+P_{\mathrm{FA}}\right] t_{\mathrm{I}}
$$

where $t_{\mathrm{D}}$ is the time that a packet acquired from any of the $S_{\mathrm{D}}$ stations keeps the destination busy and $t_{\mathrm{I}}$ is the equivalent of $t_{\mathrm{D}}$ for the $S_{\mathrm{I}}$ and $I$ stations. Note that $t_{\mathrm{I}}<t_{\mathrm{D}}$ (see Section III for their numerical values). The probability of false alarm $P_{\mathrm{FA}}$ is expressed as

$$
P_{\mathrm{FA}}=\frac{\lambda\left(1-P_{\text {busy }}\right) \Theta I}{\lambda_{0}\left(S_{\mathrm{D}}-1\right)+\lambda p_{\text {acq }} S_{\mathrm{I}}+\lambda\left(1-P_{\text {busy }}\right) \Theta I}
$$

where $\Theta$ is a parameter that depends on the particular acquisition method used. The probability of false alarm $P_{\mathrm{FA}}$ is the probability that the destination detects and acquires a packet on a different acquisition sequence (assuming it is not busy). Since equation (11) also depends on $p_{\text {acq, }}$, we must solve a quadratic system of equations composed of equations (9), (11) and (12) in order to obtain $P_{\text {busy }}$ and $p_{\text {acq }}$.

2) Computing $Q(l)$ : the Transmission Markov Chain: In order to compute $Q(l)$, we model the behavior of a station transmitting a packet with the discrete-time, homogeneous Markov chain $Z_{n}$. Let $L_{p}$ be the number of chips per packet. Since our model must take into account the fact that a source can only transmit one packet at a time, $Z_{n}$ has $L_{p}+1$ states; state 0 is the idle state where no packet transmission occurs, the states 1 to $L_{p}$ are the states where a packet transmission is happening (see Figure 1, right).

The transition probabilities of $Z_{n}$ are

$$
\left\{\begin{array}{l}
p_{Z}(0,0)=1-q \\
p_{Z}(0,1)=q \\
p_{Z}(i, i+1)=1, \quad i=1, \ldots, L_{p} \\
p_{Z}\left(L_{p}, 1\right)=q \\
p_{Z}\left(L_{p}, 0\right)=1-q
\end{array}\right.
$$


where $q$ is the probability that a packet transmission starts. The stationary distribution of $Z_{n}$ is

$$
\begin{aligned}
& \pi_{Z}(0)=\frac{1-q}{1+q\left(L_{p}-1\right)} \\
& \pi_{Z}(i)=\frac{q}{1+q\left(L_{p}-1\right)}, \quad i=1, \ldots, L_{p}
\end{aligned}
$$

In order to properly relate $\lambda$ with $q$, let $N_{p}$ be the number of packet transmitted during a time interval $t$. Since $N_{p}=\lambda t$, we have $\pi_{Z}(1)=\frac{N_{p}}{t}=\lambda$. Therefore, using (15) for $i=1$ we obtain

$$
q=\frac{\lambda}{1-\lambda\left(L_{p}-1\right)}
$$

3) Probability of Packet Transmission during Acquisition: Formally, we have

$$
\begin{aligned}
Q(l) & =\mathbb{P}(\text { A source does not visit state } 1 \text { in }[0, l-1]) \\
& =\mathbb{P}\left(X_{0} \neq 1, X_{1} \neq 1, \ldots, X_{l-1} \neq 1\right)
\end{aligned}
$$

In addition, let

$$
Q(l \mid i)=\mathbb{P}\left(X_{0} \neq 1, X_{1} \neq 1, \ldots, X_{l-1} \neq 1 \mid X_{0}=i\right)
$$

Hence, $Q(l)=\sum_{i=0}^{L_{p}} Q(l \mid i) \pi_{Z}(i)$. Now, by definition

$$
Q(0 \mid i)= \begin{cases}0 & \text { if } i=1 \\ 1 & \text { otherwise }\end{cases}
$$

and by construction

$$
Q(l \mid i)= \begin{cases}0 & \text { if } i=1 \\ \sum_{j \neq 1} p_{Z}(i, j) Q(l-1 \mid j) & \text { otherwise }\end{cases}
$$

Now, let $\vec{y}_{l}=\left[\begin{array}{llll}Q(l \mid 0) & Q(l \mid 1) & \ldots & Q\left(l \mid L_{p}\right)\end{array}\right]^{T}$. We have

$$
\overrightarrow{y_{l}}=A \vec{y}_{l-1}=A^{l} \vec{y}_{0}
$$

where $\vec{y}_{0}=\left[\begin{array}{lllll}1 & 0 & 1 & \ldots & 1\end{array}\right]^{T}$ and $A$ is equal to the transition matrix of the transmission Markov chain, except for the elements of the second row and second column that are set to 0, i.e.

$$
A(i, j)= \begin{cases}0 & \text { if } i=1 \text { or } j=1 \\ p_{Z}(i, j) & \text { otherwise }\end{cases}
$$


Finally, thanks to the structure of $A$, Equation (19) becomes

$$
\overrightarrow{y_{l}}=\left[\begin{array}{c}
(1-q)^{l} \\
0 \\
(1-q)^{\max \left(0, l-L_{p}+2\right)} \\
\vdots \\
(1-q)^{\max (0, l)}
\end{array}\right]
$$

Using the results of this section and of Section II-B, we obtain $\lambda_{0}$ by solving (1) numerically.

\section{Performance Evaluation}

For the ns-2 [5] simulations, we use the DCC-MAC layer protocol on top of an IR-UWB physical layer. DCC-MAC is described in [1] along with the model of multi-user interference used in our ns-2 implementation. We assume the use of pseudo-random time-hopping sequences.

For this paper, we have implemented additional code at the physical layer to properly model the effect of packet detection and timing acquisition. The code is available online at [8]. When a packet arrives at a destination, all further packets arriving during the duration of the acquisition sequence are stored in a list. At the end of the duration of the acquisition sequence, a packet in the list is chosen randomly (with a uniform distribution). This packet is further received by the physical layer with a probability $1-P_{\mathrm{MD}}$. If a private acquisition sequence is used, we only add to the list the packets intended for the destination. We add packets with a different acquisition sequence to the list with a probability $\Theta$ (see (12)). In the case of a common destination, we add all packets arriving during the duration of the acquisition sequence to the list.

The parameters of DCC-MAC have been adapted for an 802.15.4a type of network. In particular, the maximum physical layer rate is $1 \mathrm{Mbit} / \mathrm{s}$ and the maximum range is about 50 meters. Values for $P_{\mathrm{MD}}$ (in (10)) are derived from [3], and values for $\Theta$ (in (12)) are found by matching (12) to $P_{\mathrm{FA}}$ in [3]. For both UDP and TCP, the payload size is 1000 bytes. For the scenarios with multi-hop forwarding, we use static routing.

The throughput is given in kbit/s; given the payload $P_{\text {packet }}$ of a packet in bit, the throughput is simply $\lambda_{0} P_{\text {packet }}$.

The code for the fixed point problem has been implemented in Matlab. For the parameters of equation (8), we have the following values: 
- $t_{\mathrm{acq}}$ is the propagation time plus the length of the acquisition sequence. According to [3], the duration of the acquisition sequence is $64000 \mathrm{~ns}$.

- The transmission time $t_{\mathrm{tx}}$ is the round-trip time plus the DATA packet duration, the ACK packet duration, the SIGIDLE packet duration (see [1]) and the maximum backoff time.

- The time in case of failure $t_{\text {fail }}(i)$ is the sum of the send timer, the idle timer (see [1]) and the average backoff time in backoff stage $i$.

- In case of a packet drop, $t_{\text {drop }}$ is the maximum backoff timer length.

The detailed values can be found in [1] and [8]. Note that in the ns-2 implementation, we only drop packets after full reception.

In case of early discard, $t_{\mathrm{I}}$ (see (11)) is equal to the duration of an ACK packet transmission. For late discard, $t_{\mathrm{I}}$ is equal to a DATA packet transmission. The parameter $t_{\mathrm{D}}$ is equal to the duration of a DATA packet transmission plus an ACK packet transmission.

\section{A. Saturation Throughput of a Homogeneous UWB Network}

On Figure 2, we validate our analytical model with ns-2 simulations with UDP traffic. We take a distance of 10 meters between sources and their destination for $t_{\text {prop }}$. We have one and two destinations with $n$ sources each. As it can be observed, in both cases there is a slight discrepancy when the number of transmitters is small.

On Figure 3, we display the analytical saturation throughput in three scenarios: one destination, two destinations and eight destinations. There are $n$ sources per destination. For each case, we display the saturation throughput versus $n$ with a common acquisition sequence. The results with the private acquisition sequence are not shown since they almost overlap with the results with one destination. Note that the throughput is notably increased when dropping unintentionally acquired packets after the header. On the other hand, the throughput suffers a lot when all sources use the same acquisition sequence.

We now turn to ns-2 simulations an evaluation in more realistic scenarios. For all results obtained with ns- 2 simulation, the confidence intervals are the $95 \%$ confidence interval for the median. 


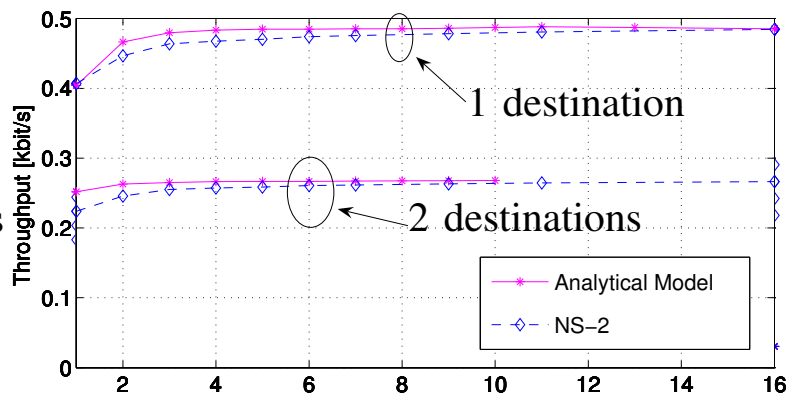

Fig. 2. Validation of the results obtained with the analytical model. The aggregated saturation throughput is plotted (sum of all sources) versus the number of sources per destination $n$. The plain curve is the analytical saturation throughput, the dashed curve is the ns-2 simulations. The upper curves are for one destination with $n$ transmitters. For the bottom curves, there is a second destination with $n$ concurrent transmitters using the same acquisition sequence.

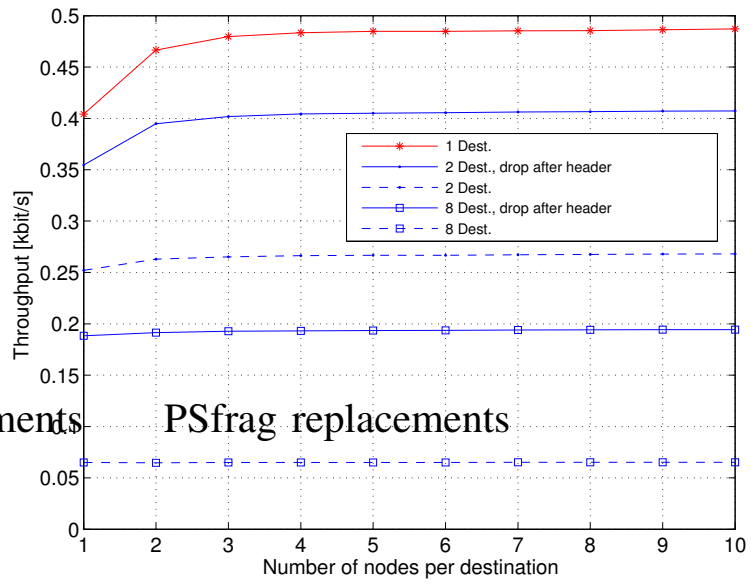

Fig. 3. The aggregated throughput (sum all throughputs) is plotted versus the number of nodes per destination $n$. We have 1,2 and 8 destinations. Each time we display the saturation throughput with the common acquisition sequence. The results with the private acquisition sequence are not show since they almost overlap with the results with 1 destination. There is large drop in throughput when all sources use the same acquisition sequence.

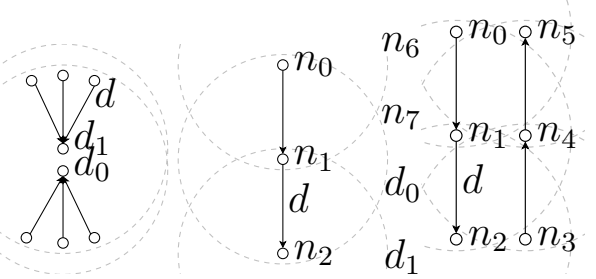

Fig. 4. Topologies for the multiple piconets scenario, for the line TCP scenario and the parallel TCP flows scenario. The link distance is $d$. 


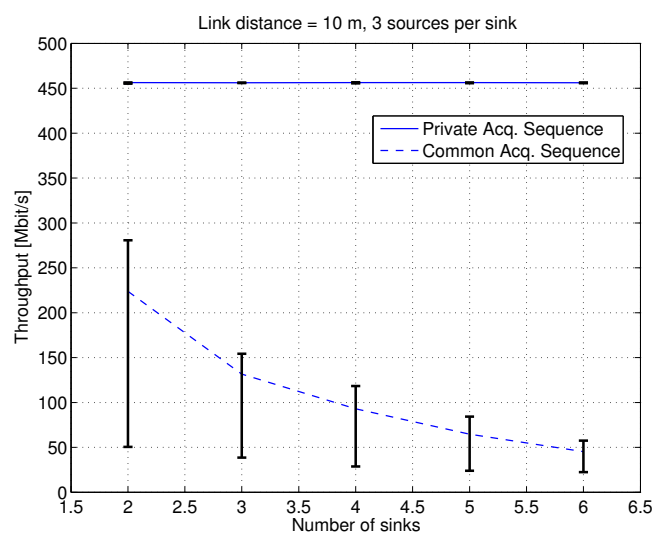

Fig. 5. Several interfering piconets (see Figure 4): saturation throughput versus number of piconets. The difference between the throughput with a private acquisition sequence and a common acquisition sequence grows with the number of interferers. In addition, the network is much more unstable when all sources use the same acquisition sequence.

\section{B. Multiple Piconets}

In this scenario, we have $n$ piconets with 3 sources and 1 destination per piconet. All nodes are in range of each other. All sources of a given piconet talk to the same destination inside the piconet. UDP is used. The distance between sources and their destination is 10 meters (see Figure 4). The distance between the respective destinations of the two piconets is 4 meters. We plot the saturation throughput versus the number of piconets. The throughput highly suffers when a common acquisition sequence is used. The difference between the throughput with a private acquisition sequence and a common acquisition sequence grows with the number of interferers.

\section{Line TCP and Parallel TCP flows}

The first scenario is a line of nodes with the sender and the destination at each extremity of the line. Multi-hop forwarding is used between the source and the destination. TCP is used as the transport protocol. In the second scenario (see Figure 4), we have two parallel lines of nodes that each run a TCP session between the two extremities. For each line, the source and destination are inverted. For the line TCP scenario the link distance $d$ is either 10 or 20 meters. For the parallel scenario, the link distance is 20 meters and the distance between the two lines is 20 meters. For both cases, we show the throughput as a function of the number of nodes. For the parallel case, we show the result for the two flows separately. 


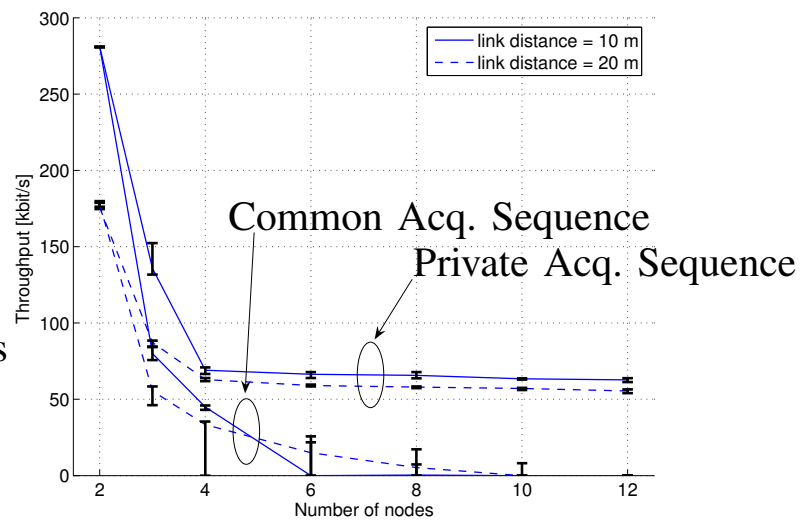

Fig. 6. Line TCP scenario: throughput versus number of nodes. This scenario shows a dramatic compounding effect where the throughput in case of a common acquisition sequence drops to zero for more than 6 nodes. The network is also much more unstable when all sources use the same acquisition sequence.

In the line TCP scenario (Figure 6), we observe a dramatic compounding effect when all sources use the same acquisition sequence. For more than 6 destinations, the throughput reaches zero when using the common acquisition sequence. In addition there is much more variability in the network behavior than when using private acquisition sequences.

An even more severe effect is observed when using a common acquisition sequence in the case of the two parallel TCP flows (Figure 7). In addition to an almost complete collapse of the network when the number of nodes is large than six (i.e. more than two hops), we observe a high unfairness between the two flows. This behavior is very similar to what can happen in 802.11 networks in the exposed node case [9] and this, even though we are using a multi-user physical-layer.

\section{CONCLUSION}

We have analyzed the effect on the network performance of using a private or common acquisition sequence. For several scenarios, we show a very large throughput increase (>100\%) when using a private acquisition sequence. This throughput increase largely justify the use of a private acquisition sequence even though there is a cost in learning the acquisition sequence. The throughput difference grows with the number of concurrent transmitters and interferers. We also show the presence of a compounding effect similar to exposed terminal issues in 802.11 networks. Further, the use of a common acquisition sequence provokes very large performance 


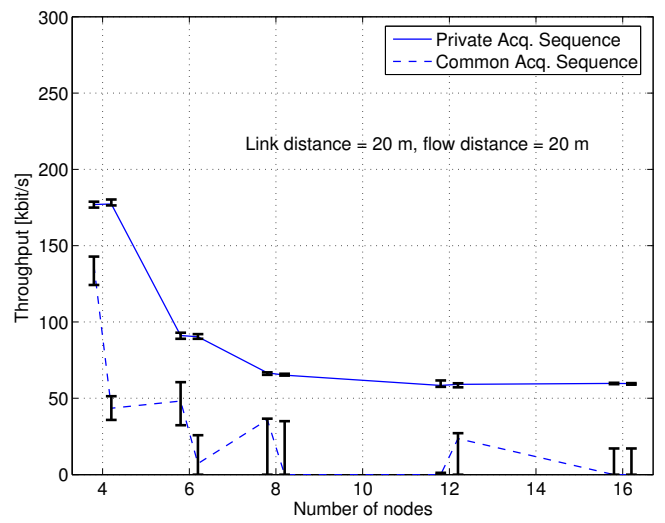

Fig. 7. Parallel TCP flows scenario: throughput per flow versus number of nodes. For each set of nodes, flow 1 is on the left, flow 2 is on the right. This scenario shows a dramatic compounding effect where the network completely collapses in case of a common acquisition sequence for more than two hops. There is also a high unfairness between the two flows.

fluctuations in some scenarios. Future work will analyze the cost of learning the acquisition sequence of a destination.

\section{REFERENCES}

[1] R. Merz, J. Widmer, J.-Y. Le Boudec, and B. Radunovic, "A joint PHY/MAC architecture for low-radiated power TH-UWB wireless ad-hoc networks," Wireless Communications and Mobile Computing Journal, Special Issue on Ultrawideband (UWB) Communications, vol. 5, no. 5, pp. 567-580, August 2005.

[2] M.-G. Di Benedetto, L. Nardis, M. Junk, and G. Giancola, “(UWB)² : Uncoordinated, wireless, baseborn, medium access control for UWB communication networks," Mobile Networks and Applications, vol. 10, no. 5, October 2005.

[3] A. El Fawal and J.-Y. Le Boudec, "A robust signal detection method for ultra wide band (UWB) networks with uncontrolled interference," IEEE Transactions on Microwave Theory and Techniques, 2006, to appear.

[4] G. Bianchi, "Performance analysis of the IEEE 802.11 distributed coordination function," IEEE Journal on Selected Areas in Communications, vol. 18, no. 3, pp. 535-547, March 2000.

[5] "The network simulator ns-2, http://www.isi.edu/nsnam/ns/."

[6] C. Bordenave, D. Mc Donald, and A. Proutière, "Random multi-access algorithms, a mean field analysis," in Forty-third annual Allerton conference on Communication, Control, and Computing, 2005.

[7] F. Baccelli and P. Brémaud, Palm Probabilities and Stationary Queues. Springer Verlag Lecture Notes in Statistics, March 1987.

[8] "UWB research at EPFL-IC," http://icawww1.epfl.ch/uwb/, 2006.

[9] K. Xu, M. Gerla, L. Qi, and Y. Shu, "Enhancing TCP fairness in ad hoc wireless networks using neighborhood RED," in MobiCom '03, 2003, pp. 16-28. 